\title{
Cooperative Optimization QoS Cloud Routing Protocol Based on Bacterial Opportunistic Foraging and Chemotaxis Perception for Mobile Internet
}

\author{
Shujuan Wang, ${ }^{1}$ Long He, ${ }^{2}$ and Guiru Cheng ${ }^{1}$ \\ ${ }^{1}$ Changchun University of Technology, Changchun 130012, China \\ ${ }^{2}$ Sinopharm A-THINK Pharmaceutical Co. Ltd., Changchun 130012, China \\ Correspondence should be addressed to Guiru Cheng; chengguiru@ccut.edu.cn
}

Received 29 September 2015; Accepted 29 November 2015

Academic Editor: James Nightingale

Copyright (c) 2015 Shujuan Wang et al. This is an open access article distributed under the Creative Commons Attribution License, which permits unrestricted use, distribution, and reproduction in any medium, provided the original work is properly cited.

In order to strengthen the mobile Internet mobility management and cloud platform resources utilization, optimizing the cloud routing efficiency is established, based on opportunistic bacterial foraging bionics, and puts forward a chemotaxis perception of collaborative optimization QoS (Quality of Services) cloud routing mechanism. The cloud routing mechanism is based on bacterial opportunity to feed and bacterial motility and to establish the data transmission and forwarding of the bacterial population behavior characteristics. This mechanism is based on the characteristics of drug resistance of bacteria and the structure of the field, and through many iterations of the individual behavior and population behavior the bacteria can be spread to the food gathering area with a certain probability. Finally, QoS cloud routing path would be selected and optimized based on bacterial bionic optimization and hedge mapping relationship between mobile Internet node and bacterial population evolution iterations. Experimental results show that, compared with the standard dynamic routing schemes, the proposed scheme has shorter transmission delay, lower packet error ratio, QoS cloud routing loading, and QoS cloud route request overhead.

\section{Introduction}

Research of QoS (Quality of Services) guarantees that routing protocol has been the focus issue in mobile Internet [1]. In particular, some research staff has combined the bionics research results of chemotaxis system [2,3] and bacterial foraging optimization [4] with the above problem. However, how to improve the QoS routing protocol effect of mobile Internet and Cloud platform has not been in-depth study.

On the one hand, Szymanski [5] presented a Constrained Multicommodity Maximum-Flow-Minimum-Cost routing algorithm, which could compute the maximum-flow routings for all smooth unicast traffic demands within the Capacity Region of a network subject to routing cost constraints. Berger et al. [6] proposed the interdomain traffic routing for dealing with the generalization of the shortest path and path-trading problem. A model of Cascading Failures on the Interdomain Routing System was proposed by Liu et al. [7]. The Virtual Internet Routing Lab platform was shown in article [8], which was used in the training, education, or research of Internet service. A delay-guaranteed energy profile-aware routing (DEAR) algorithm proposed for a green Internet was proposed to resolve the limitations of energy profiles and delay guarantees in article [9].

On the other hand, a variant of the bacterial foraging optimization algorithm was proposed by Tan et al. [10], which included the time-varying chemotaxis step length and comprehensive learning strategy. Naveen et al. [11] formulated the network reconfiguration problem as nonlinear objective optimization problem. A new long term scheduling for optimal allocation of capacitor bank in radial distribution system was presented by Devabalaji et al. [12], which can minimize the system power loss to resolve the equality constraints. The quantitative behavior of one-dimensional classical solutions for a hyperbolic-parabolic system describing repulsive chemotaxis was investigated in article [13]. Based on the knowledge of fuzzy mathematics, microeconomics, and swarm intelligence, the author proposed the flexible QoS 
unicast routing scheme with QoS satisfaction degree and utility introduced [14]. The novel bacterial chemotaxis optimization method (BCO) to QoS multicast routing scheme was proposed by Yong [15]. Chen et al. [16] proposed the bacterial colony optimization algorithm based on bacterial chemotaxis.

On the basis of the above research results, we studied the bacterial foraging model of mobile Internet and presented the chemotaxis perception cooperative routing protocol. Finally, the QoS cloud routing protocol for mobile Internet was proposed.

The rest of the paper is organized as follows. Section 2 describes the bacterial foraging model. In Section 3, we design the chemotaxis perception cooperative routing protocol. In Section 4, we proposed the QoS cloud routing protocol for mobile Internet. Experiment results are given in Section 5. Finally, we conclude the paper in Section 6.

\section{Bacterial Foraging Model for Internet}

In the mobile Internet, we set each mobile node as a bacterium. The behavior of mobile nodes searching for the next hop data receiving nodes is defined as bacterial foraging. Mobile nodes movement process is defined as a bacterial motility. When $k$ bacterium is moving, the target was searching in the subnetwork of current bacteria. Set the distance to the $d_{b k}$. Then, the different directions of the current crawling angle as $\alpha$ were chosen. When $k$ bacterium is in the direction of the movement direction of the best individual in the subnetwork, the attraction force of the bacteria $b_{\mathrm{af}}$ is determined by the following formula:

$$
\begin{aligned}
d_{b k} & =\frac{t_{k} \sqrt{v_{k} \sin \alpha}}{\tan \beta}, \\
b_{\mathrm{af}} & =\left[b_{p}-\sum_{i=1}^{k} \frac{\sin \beta}{d_{b k}}\right]^{-\cos \alpha} .
\end{aligned}
$$

Here, $t_{k}$ denotes the peristalsis delay of $k$ bacteria. $v_{k}$ denotes the peristalsis speed of $k$ bacteria. $b_{p}$ denotes the overall dynamics of bacterial populations. $\beta$ is the angle of antenna and signal direction.

The mobile Internet is a multidimensional space field of the bacteria. Data transmission route selection problem is decomposed into the problem of the bacteria on the multidimensional field. The food delivery process may be accomplished by a number of bacteria in the same field, and it may be completed by a new structural field $S_{F}$, which is derived from the bacterial assemblage from different fields, as defined in

$$
\begin{aligned}
& V_{S_{F}}= {\left[v_{1}, v_{2}, \ldots, v_{n}\right], } \\
& S_{F}=\left[\begin{array}{c}
b_{1} \\
b_{2} \\
\vdots \\
b_{n}
\end{array}\right] V_{S_{F}} .
\end{aligned}
$$

Here, $V_{S_{F}}$ is the bacterial movement velocity in the structure field. $v$ is the mobile speed of node. $b$ is the amount of food carried by the bacteria. The amount of information transmitted is denoted by $S_{F}$ for the mobile communication of the structure field.

The dynamic changes of the multifield and the structure of the bacteria in the process of the movement and feeding of the bacteria are shown in Figure 1. There is bacteria foraging in multidimensional field, when the same field can not meet the needs of food delivery and other fields through mapping to establish the route, thus generating the structure, and there are various fields to select the best bacteria to join the structure of the field. By this mapping mechanism, it can prevent the local best bacterial ability to limit the population creep performance but also can shorten the time of route update and improve the convergence precision of the searching. The mapping results could be connected with the Internet by combining with the wireless cooperative networks.

Among them, from the dynamic evolution of the multidimensional field to the structural field, the principle of bacterial opportunity to feed is used. In Figure 3, three multidimensional fields and two structural fields are given. When the bacteria foraging from $M_{F 1}$ and $M_{F 2}$ to $M_{F 3}$ could not satisfy the transmission requirements, the opportunity structure field would be constructed based on the optimal direction and speed of the multidimensional field. Therefore, there is the different bacteria structure, the food principle, and the food amount. For example, $S_{F 1}$ is composed of good bacteria from $M_{F 1}$ and $M_{F 2}$. The food principle is the XOR operation between $M_{F 1}$ and $M_{F 2}$. The food amount is the sum of the food scales of $M_{F 1}$ and $M_{F 2}$. $S_{F 2}$ would be obtained by the fusion of $S_{F 1}$ and $M_{F 1}, M_{F 2}$, and $M_{F 3}$. The bacteria in the field are dynamic, which could be selected and optimized according to the requirements of food and information transmission requirements in the multidimensional field. The structural parameters of the above structures are shown in the following formula:

$$
\begin{aligned}
b_{k}^{t} & =b_{k}\left(\alpha, \beta, b_{k}\right), \\
S_{F 1} & =\operatorname{sub}\left(M_{F 1}\right) \cup \operatorname{sub}\left(M_{F 2}\right) \cup \operatorname{sub}\left(M_{F 3}\right) \sum_{I=1}^{K} b_{k}^{t}, \\
S_{F 2} & =\delta\left(M_{F 1} \cap M_{F 2} \cap M_{F 3}\right) \cup \operatorname{sub}\left(M_{F}\right) \cup S_{F 1} .
\end{aligned}
$$

Here, let $b_{k}^{t}$ denote transfer peristalsis from the multidimensional field to the structural field of $k$ bacteria. $\delta$ is opportunity foraging factor of bacteria, which is determined by the bacteria in real time and the attraction force.

Hence, the bacteria opportunity foraging algorithm in the mobile Internet described is as follows.

Algorithm $1\left(\mathrm{BOF}\left(d_{b k}, b_{\mathrm{af}}, \alpha, \beta, \delta\right)\right)$.

BOF function Initialization of each Bacteria

$\alpha$ and $\beta$ to $d_{b k}$

$b_{p}$ to $b_{\mathrm{af}}$

Searching for optimal bacteria in $M_{F}$ 


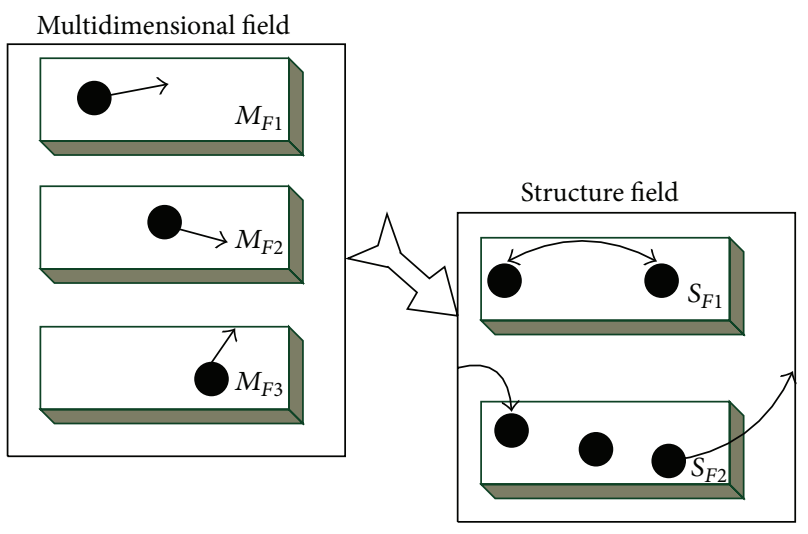

FIGURE 1: Multidimensional field and structure field of bacterial opportunistic foraging.

$$
\begin{aligned}
& \text { For } i=1 \text { to } k \\
& \{ \\
& \text { Statistics } V_{S_{F}} ; \\
& \text { Compute } S_{F} ; \\
& S_{F 1} \leftarrow \operatorname{sub}\left(M_{F 1}\right) \cup \operatorname{sub}\left(M_{F 2}\right) \cup \operatorname{sub}\left(M_{F 3}\right) \sum_{I=1}^{K} b_{k}^{t} ; \\
& \left.S_{F 2} \leftarrow \delta\left(M_{F 1} \cap M_{F 2} \cap M_{F 3}\right) \cup \operatorname{sub}\left(M_{F}\right) \cup S_{F 1}\right\} \\
& \operatorname{return} S_{F}
\end{aligned}
$$

\section{Chemotaxis Perception Cooperative Routing Protocol}

In the process of bacterial foraging, the direction of bacterial motility and the structure of the field are characterized by chemotaxis. All the bacteria in the field can creep along the same direction and can also be spread to the food in the field of structural field after the food is carried and dispersed. The position of a trend of drug resistance is shown in the following formula:

$$
b_{k}^{i+1}=b_{k}^{i}\left(\alpha, \beta, b_{f}\right)+\frac{\sum_{i=1}^{L} S_{F_{i}}}{\sqrt{\Delta S_{F}}} .
$$

Here, $\Delta S_{F}$ is the update information of the structure field.

On the basis of chemotaxis perception, the behavior of the bacterial foraging population has the characteristics of dormancy, activation, and direction. When bacteria are crawling, the bacteria are perceived to be more resistant and opportunistically release the lure of the bacteria to the neighbor and move along the structural field for the next round. When the bacteria in the active state are all completed by the lure and the movement, the whole structure field becomes an active field, and the relationship between the bacteria and the colony behavior of the two groups is shown in the following formula:

$$
\begin{aligned}
B_{S_{F}}\left(b_{k}, n, m\right) & =\sum_{i=1}^{n} b_{k}^{m} B_{S_{F}}^{i}\left(b_{k}^{i}, i, m\right), \\
B_{S_{F}} & =\sum_{j=1}^{m}\left[-\gamma \exp \left(\cos (|\alpha-\beta|)^{2}\right)\right]^{m-j} .
\end{aligned}
$$

Here, $B_{S_{F}}$ denotes the bacterial population behavior of structure field. $n$ is bacterial number of structure fields. $m$ is the number of neighbors who have become more resistant to the bacteria. With the bacterial motility and structural field of the update, the attraction of the drug is gradually enhanced. Bacterial colony behavior becomes gradually mature. The position and direction of the bacteria are unified, as shown in the following formula:

$$
\begin{aligned}
B_{S_{F}}^{i+1}\left(b_{k}, i+1, m\right) & =\cos (|\alpha-\beta|)^{2} B_{S_{F}}^{i}\left(b_{k}^{i}, i, m\right), \\
b_{k}^{i+1} & =b_{k}^{i}(\sin \alpha, \arctan \beta, \gamma) .
\end{aligned}
$$

After multiple iterations of the search, the driving of the chemotaxis and the total number of bacteria in the food are shown in the following formula:

$$
T_{\mathrm{CH}}\left(B_{S_{F}}^{n}\right)=\sum_{i=1}^{n} b_{p}^{i} \exp \left(\sin ^{2} \alpha-\arctan ^{2} \beta\right) .
$$

Here, $T_{\mathrm{CH}}$ denotes the total foraging amount of bacteria population. In order to make full use of the characteristics of the drug resistance to the bacteria, the mobile node route is established, and the angle parameters of the bacterial motility and the attraction force are calculated.

At the same time, in order to speed up the accumulation of bacteria and route establishment, the bacterial community would be sorted linearly and opportunistically according to the food principle and individual food amount. The bacteria with good performance would be produced through the drug resistance drive. In order to simplify the algorithm, in order to maintain the vitality of the population, the bacteria and the bacteria in the dormant state are consistent with the direction of the movement of the drug.

In the actual Internet communication environment, the transmission data of mobile nodes is affected by external forces or their resources are limited, and the process of routing communication based on bacterial chemotaxis may be destroyed. But the bacterial population is helping to find a more abundant area of food. Therefore, in view of the endto-end communication, the mechanism of the drug delivery mechanism based on the bacterial cooperative control is beneficial to the bacterial foraging and population stability. In the cooperative routing algorithm, the individual behavior and the behavior of the bacteria are repeated after many iterations, and the bacteria can be a certain probability to the recombinant population or the structure field and will be the advantage of the spread of the food gathering area. The mobile node information fusion scheme can not only protect 
TABLE 1: Internet settings.

\begin{tabular}{lccc}
\hline Parameter & Value & Parameter & Value \\
\hline Simulation time & $2500 \mathrm{~s}$ & Perceived distance of mobile node & $200 \mathrm{~m}$ \\
\hline Internet area & $100 \mathrm{~m} * 1200 \mathrm{~m}$ & Number of maximum active nodes & 30 \\
\hline Concurrent service number & 5 & Packet size & 1200 bytes \\
\hline Cloud number & 8 & Packet sending speed & 3 packets/s \\
\hline Disk size of cloud & 1 Tbytes & CPU number of cloud & 4 \\
\hline Kernel operating frequency & $2 \mathrm{GHz}$ & Memory size of cloud & $4 \mathrm{Gbytes}$ \\
\hline
\end{tabular}

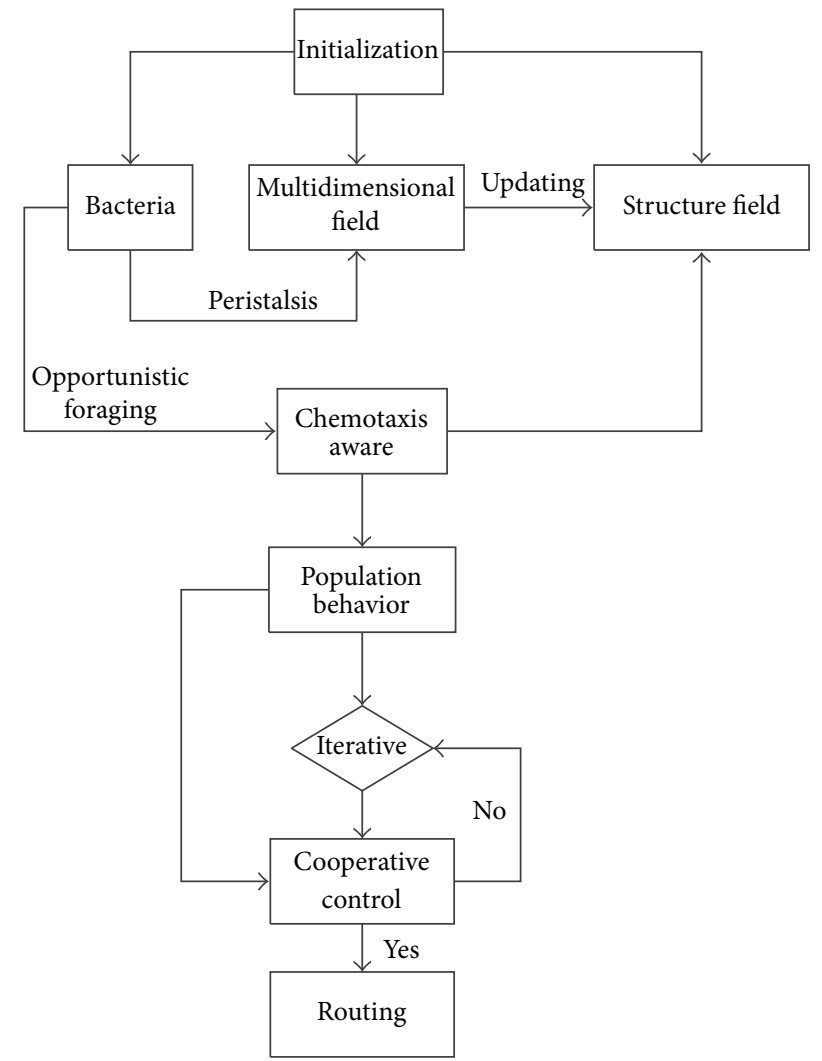

FIGURE 2: Route establishment based on bacterial opportunity to search for food and drug delivery.

the bacteria from the local optimal value but also enhance the global routing performance.

In summary, the establishment of a route based on bacterial opportunity foraging and the route of drug delivery is described in Figure 2.

\section{QoS Cloud Routing Protocol for Mobile Internet}

In the mobile Internet to join the cloud platform, the mobile node's bacterial opportunity foraging behavior can express the communication distance between the data sending node and the receiving node. Bacterial chemotaxis of mobile nodes can reflect the quality and stability of the communication link between the Internet and the cloud platform. In the actual communication environment, by detecting the degree of attenuation of the bacterial foraging ability of the mobile nodes, the population behavior of the mobile node is analyzed, such as the direction and speed of the short time of the mobile node, and the probability of the interruption of the link is caused by the structural field update. In the cloud routing mechanism of QoS, the opportunistic optimization of the bacterial population is formed, which will lead to a large loss of multipath gain between the Internet and the cloud platform. Therefore, QoS cloud routing bacterial bionic optimization is based on multipath by establishing mechanism through hedge mapping between iterations in mobile Internet node physical layer of the antenna array and bacterial population evolution. QoS cloud routing path was selected and optimized.

The signal in the end-to-end communication with the bacterial opportunity foraging is shown in the following formula:

$$
\operatorname{Sig}_{b}=\sqrt{h_{t}^{2} h_{r}^{2}} \frac{\sin ^{\sqrt{G_{t} G_{r}}} \alpha}{b_{d k}^{n}} .
$$

Here, $G_{t}$ denotes antenna gain of sending node. $G_{r}$ denotes antenna gain of receiving node. $h_{t}$ and $h_{r}$ denote the antenna height of the transmitter and the receiving nodes, respectively. If $b_{d k}^{n}$ is larger than 1 , the bacterial opportunity is not related to the attraction force of the population. In the QoS cloud routing request message in the bacterial population in the process, the activation of the neighbor bacteria opportunity type according to the movement speed and load information of the opportunity to calculate the cloud path of the opportunity weight $W$ is shown in the following formula:

$$
w_{k}=\prod_{k \in S_{F}}\left(\delta \frac{\rho-b_{d k}}{\rho}\right) \tan \beta .
$$

Here, the evolution of the bacteria will occur, which belong to the same field. $\rho$ denotes the peristalsis bacteria foraging radius of node.

As shown in Figure 3, bacteria nodes sent out a signal of the Internet data transmitting. After the opportunity for foraging peristalsis two multidimensional cloud paths are formed in the cloud platform. By formula (9), the opportunity weights of two multidimensional field cloud paths are $w 1$ and $w 2$. Here, $w 1$ is less than $w 2$. Although number of active bacteria nodes of dotted cloud routing path is less than one of the solid cloud path, the solid cloud path has more attractive 


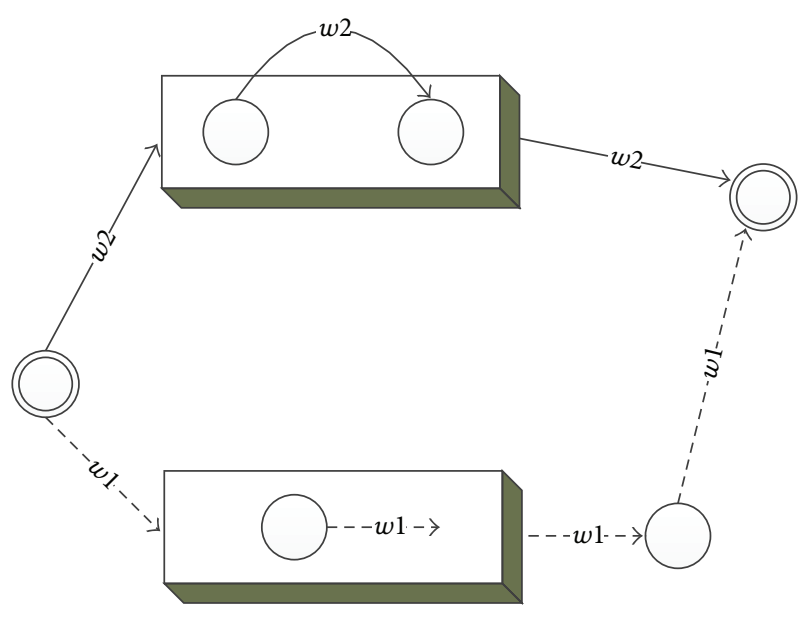

FIgURE 3: QoS cloud path selection.

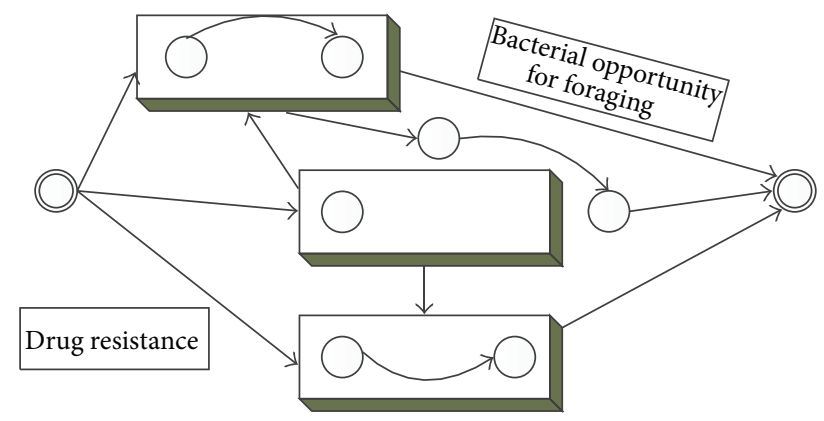

FIGURE 4: Process of QoS cloud route discovery.

force. In order to avoid breaking the path of the cloud, the current QoS cloud path should be the solid path.

QoS cloud path of cooperative maintenance process is shown in Figure 4. The RREP Format in DSR (Dynamic Source Routing) Options Header is shown in Figure 5. The bacterial neighbor node receives the request message from the data sending end node to the cloud path request message, and the bacteria spread the cloud path request to the bacteria population in the same multidimensional field through the opportunistic feed. There are multiple paths of mobile nodes in the bacterial population through collaborative construction, to maintain the cloud path through the computer.

In order to eliminate bacterial opportunity to feed into the dead cycle, the cooperative maintenance of the QoS cloud path requires the maintenance of the individual in the end to end cloud path of the chemotaxis and motility of the bacterial node sequence, to maintain its monotonic increasing characteristics, as shown in the following formula:

$$
\begin{aligned}
\left(-S_{F_{i+1}}, B_{S_{F}}^{i}\left(b_{k}^{i}, i, m\right)\right) & >\left(S_{F_{i}}, B_{S_{F}}^{i-1}\left(b_{k}^{i-1}, i-1, m\right)\right) \\
R_{b d} & =\sum_{i=1}^{L} S_{F_{i}} \frac{\cos \alpha}{\sqrt{\Delta S_{F}}} .
\end{aligned}
$$

\section{Performance Evaluation}

In this section, we analyzed and evaluated the performance of the proposed COQCRP (Cooperative Optimization QoS Cloud Routing Protocol based on bacterial opportunistic foraging and chemotaxis perception) routing protocol. Two groups of simulation experiments were designed. The DRP (Dynamic Routing Protocol) with the Internet was compared with the proposed protocol, respectively, based on the mobile speed of the nodes and the size of the Internet in the case of the QoS performance of the Internet. Table 1 records the Internet parameter settings.

In order to evaluate the performance of COQCRP, four performance evaluation criteria of QoS cloud routing are statistically analyzed.

End-to-End QoS Cloud Communication Delay. It is the time interval of data packets transmitted from the Internet node to destination node through cloud.

QoS Cloud Route Load. It is the control packet number of QoS routing cloud cooperative optimizations divided by the number of correctly received data packets.

QoS Cloud Routing Overhead. It is the number of COQCRP cloud routing requests sent or forwarded in the unit time.

In Experiment 1, the analysis of the Internet based on the mobile speed of the mobile node of the mobile speed of the QoS cloud routing performance, the results are shown in Figure 6. The meaning of coordinates $x$ and $y$ in Figure 6(a) is mobile speed of node and average end-to-end delay. The meaning of coordinates $x$ and $y$ in Figure 6(b) is mobile speed of node and packet error ratio.

The COQCRP significantly improves the end-to-end cloud communication delay, as shown in Figure 6(a). The reason is that, based on QoS cloud, opportunistic bacterial foraging by selection mechanism improves the robustness of the path of the cloud and relationship of cloud routing size for the maintenance delay. COQCRP controlled the message broadcast of cloud path request cooperatively, which improved the Internet link bandwidth utilization and avoided the cloud computing error, as well as the data packet collision probability. With the increase of the node moving speed, the performance of COQCRP and DRP is similar. This is due to the probability of packet collision and the speed of the node of the Internet is proportional to the speed of the node. In Figure 6(b), the simulation results show the effect of the change of node moving speed on the packet loss rate. With the increase of the node moving speed, the path break frequency increases and the packet loss rate increases. COQCRP data transmission path quality is improved. COQCRP can provide an effective guarantee for data packet transmission and packet error ratio is lower than DRP.

In Experiment 2, the analysis of the impact of the scale of the Internet on the QoS cloud routing performance is shown in Figure 7. The meaning of coordinates $x$ and $y$ in Figure 7 (a) is cloud scale and routing load. The meaning of coordinates $x$ and $y$ in Figure $7(\mathrm{~b})$ is cloud scale and route request overhead. 


\begin{tabular}{|c|c|c|c|}
\hline $\begin{array}{c}\text { Opportunity } \\
\text { foraging weight }\end{array}$ & $\begin{array}{c}\text { Chemotaxis } \\
\text { perception }\end{array}$ & $\begin{array}{c}\text { Length of } \\
\text { multidimensional field }\end{array}$ & $\begin{array}{c}\text { Length of } \\
\text { structure field }\end{array}$ \\
\hline \multicolumn{4}{|c|}{ Address of sending node } \\
\hline \multicolumn{3}{|c|}{ Cooperative nodes } & $4-10$ bytes $\downarrow$ \\
\hline \multicolumn{3}{|c|}{ Address of receiving node } \\
\hline
\end{tabular}

FIgURE 5: The RREP format in DSR options header.

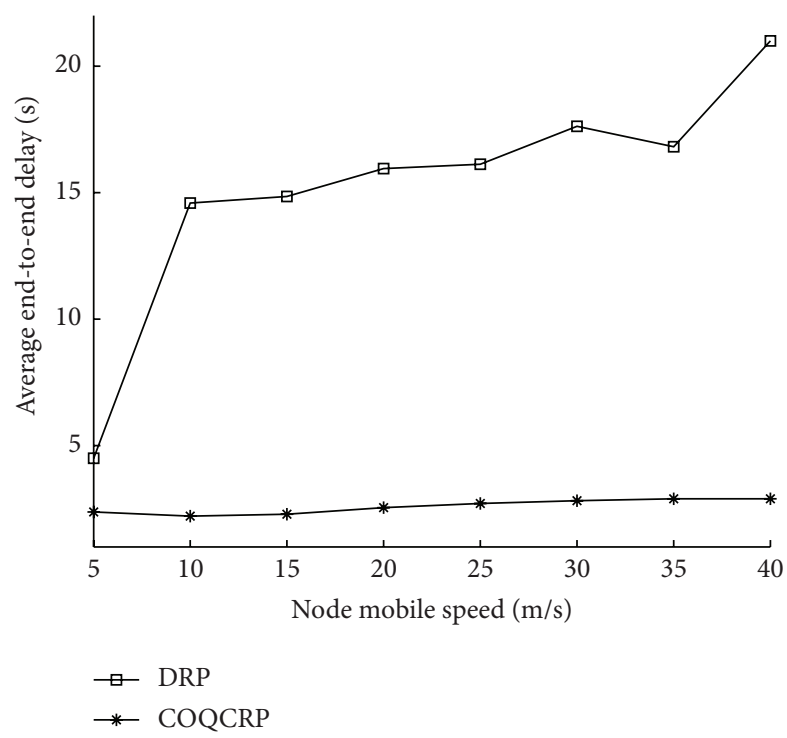

(a) Average end-to-end delay

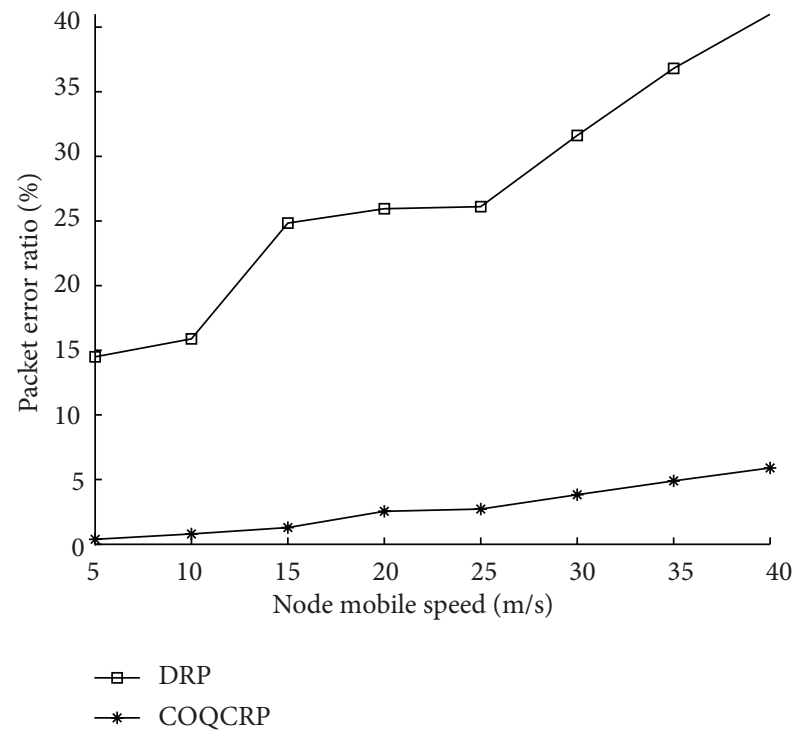

(b) Packet error ratio

FIgURE 6: Performance analysis with speed.

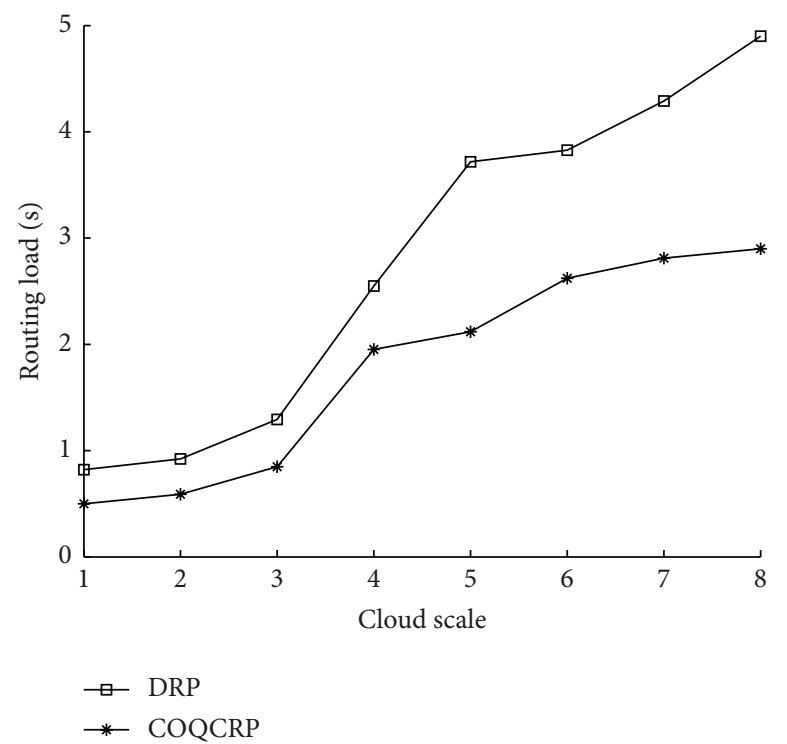

(a) Routing load

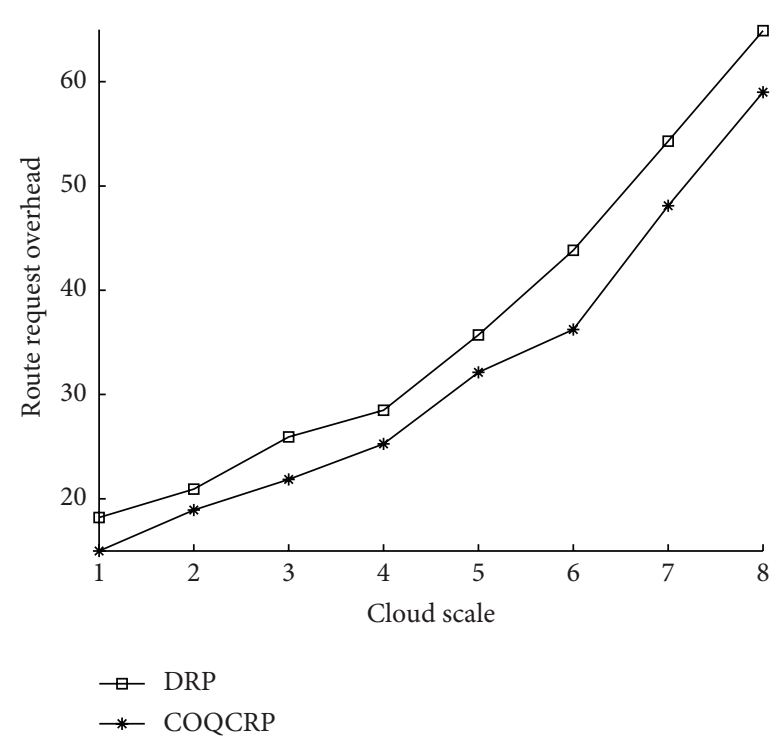

(b) Route request overhead

FIgURE 7: Performance analysis with cloud scale. 
Due to the extension of the path lifetime and COQCRP QoS cloud routing load (Figure 7(a)) and cloud routing request overhead (Figure $7(\mathrm{~b})$ ) that significantly reduced, COQCRP effectively saved the Internet resources and improved the work efficiency of the cloud platform.

\section{Conclusions}

According to the mobility management requirements of mobile Internet and cloud platform resource constrained characteristics, a chemotaxis perception cooperative QoS cloud routing optimization mechanism was proposed based on the opportunistic bacterial foraging bionics. First, we assume that each mobile node of the mobile Internet is a bacterium. A data forwarding node group was established through the progress of the bacterial opportunistic feed and the characteristic of bacterial motility. Secondly, based on the analysis of the direction of the movement of the bacteria and the characteristics of the trend of the structural field, the establishment of the mechanism of the drug resistance is established by the distributed load control of all the bacteria in the field. Finally, the cloud platform joined the mobile Internet. We combined the mobile node opportunistic bacterium foraging behavior and bacterial chemotaxis. A cooperative optimization QoS cloud routing mechanism was proposed to guarantee the quality and stability of the communication link between the Internet and cloud platform. The experimental results show that the proposed mechanism has the advantages of real-time, reliability, routing load, and resource utilization of cloud platform in QoS communication.

\section{Conflict of Interests}

The authors declare that there is no conflict of interests regarding the publication of this paper.

\section{References}

[1] M. S. Siddiqui, D. Montero, R. Serral-Gracià, X. Masip-Bruin, and M. Yannuzzi, "A survey on the recent efforts of the internet standardization body for securing inter-domain routing," Computer Networks, vol. 80, pp. 1-26, 2015.

[2] Q. S. Zhang and Y. X. Li, "Global existence and asymptotic properties of the solution to a two-species chemotaxis system," Journal of Mathematical Analysis and Applications, vol. 418, no. 1, pp. 47-63, 2014.

[3] C.-H. Huang and P. A. Iglesias, "Cell memory and adaptation in chemotaxis," Proceedings of the National Academy of Sciences of the United States of America, vol. 111, no. 43, pp. 15287-15288, 2014.

[4] I. A. Mohamed and M. Kowsalya, "Optimal size and siting of multiple distributed generators in distribution system using bacterial foraging optimization," Swarm and Evolutionary Computation, vol. 15, pp. 58-65, 2014.

[5] T. H. Szymanski, "Max-flow min-cost routing in a futureinternet with improved QoS guarantees," IEEE Transactions on Communications, vol. 61, no. 4, pp. 1485-1497, 2013.
[6] A. Berger, H. Roglin, and R. van der Zwaan, "Internet routing between autonomous systems: fast algorithms for path trading," Discrete Applied Mathematics, vol. 185, pp. 8-17, 2015.

[7] Y. J. Liu, W. Peng, J. S. Su, and Z. Wang, "Assessing the impact of cascading failures on the interdomain routing system of the internet," New Generation Computing, vol. 32, no. 3-4, pp. 237255, 2014.

[8] J. Obstfeld, S. Knight, E. Kern, Q. S. Wang, T. Bryan, and D. Bourque, "VIRL: the virtual internet routing lab," $A C M$ SIGCOMM Computer Communication Review, vol. 44, no. 4, pp. 577-578, 2014.

[9] E.-J. Lee, Y.-M. Kim, and H.-S. Park, “DEAR: delay-guaranteed energy profile-aware routing toward the green internet," IEEE Communications Letters, vol. 18, no. 11, pp. 1943-1946, 2014.

[10] L. J. Tan, F. Y. Lin, and H. Wang, "Adaptive comprehensive learning bacterial foraging optimization and its application on vehicle routing problem with time windows," Neurocomputing, vol. 151, no. 3, pp. 1208-1215, 2015.

[11] S. Naveen, K. S. Kumar, and K. Rajalakshmi, "Distribution system reconfiguration for loss minimization using modified bacterial foraging optimization algorithm," International Journal of Electrical Power and Energy Systems, vol. 69, pp. 90-97, 2015.

[12] K. R. Devabalaji, K. Ravi, and D. P. Kothari, “Optimal location and sizing of capacitor placement in radial distribution system using bacterial foraging optimization algorithm," International Journal of Electrical Power \& Energy Systems, vol. 71, pp. 383390, 2015.

[13] D. Li, R. Pan, and K. Zhao, "Quantitative decay of a onedimensional hybrid chemotaxis model with large data," Nonlinearity, vol. 28, no. 7, pp. 2181-2210, 2015.

[14] X. Wang, D. Jiang, and M. Huang, Flexible QoS Unicast Routing Scheme Based on Utility and BCC [EB/OL], Sciencepaper Online, Beijing, China, 2010.

[15] H. Yong, "QOS multicast routing algorithm based on bacterial chemotaxis optimisation," Computer Applications and Software, vol. 29, no. 11, pp. 269-271, 2012.

[16] L. Chen, L.-Y. Zhang, Y.-J. Guo, J.-Q. Zhao, and Q. Li, "Sequential blind signal separation algorithm based on bacterial colony chemotaxis," Journal on Communications, vol. 32, no. 4, pp. 7785, 2011. 

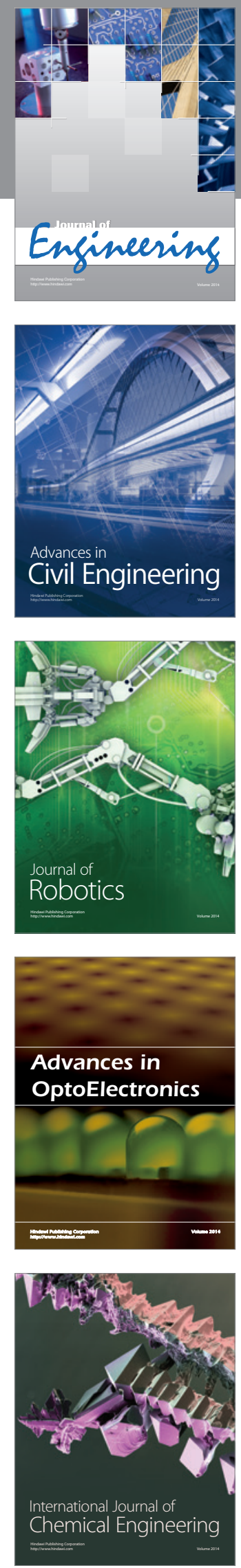

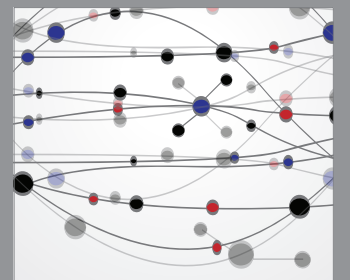

The Scientific World Journal
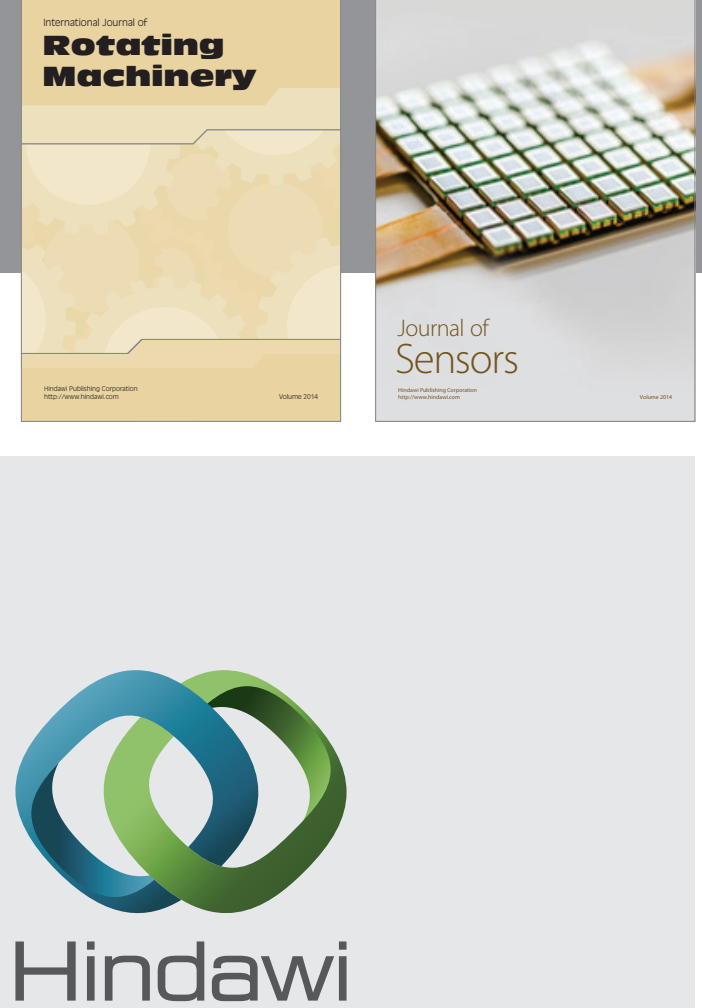

Submit your manuscripts at http://www.hindawi.com
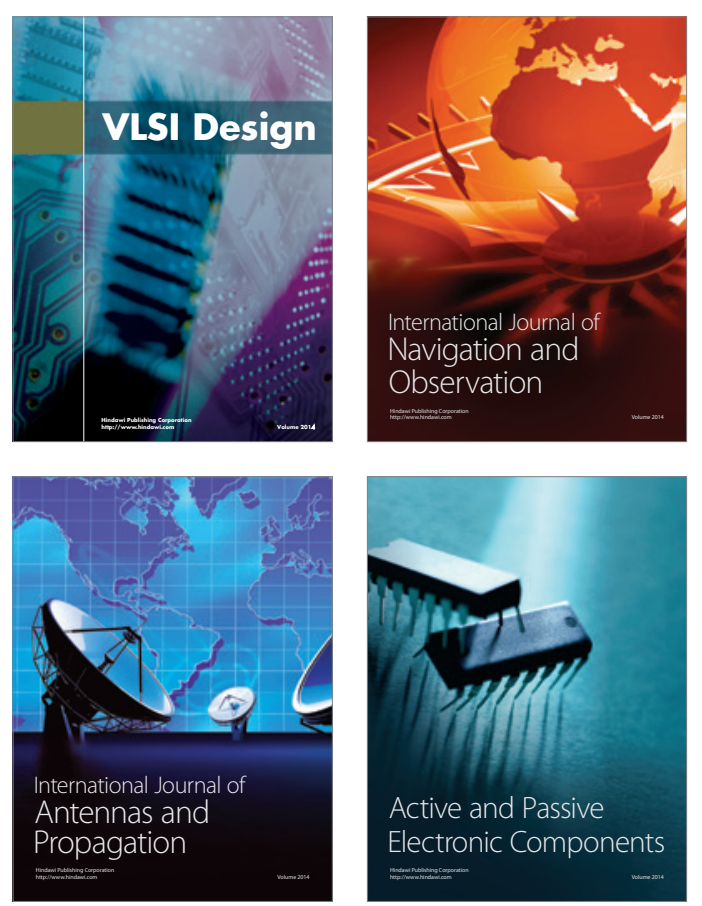
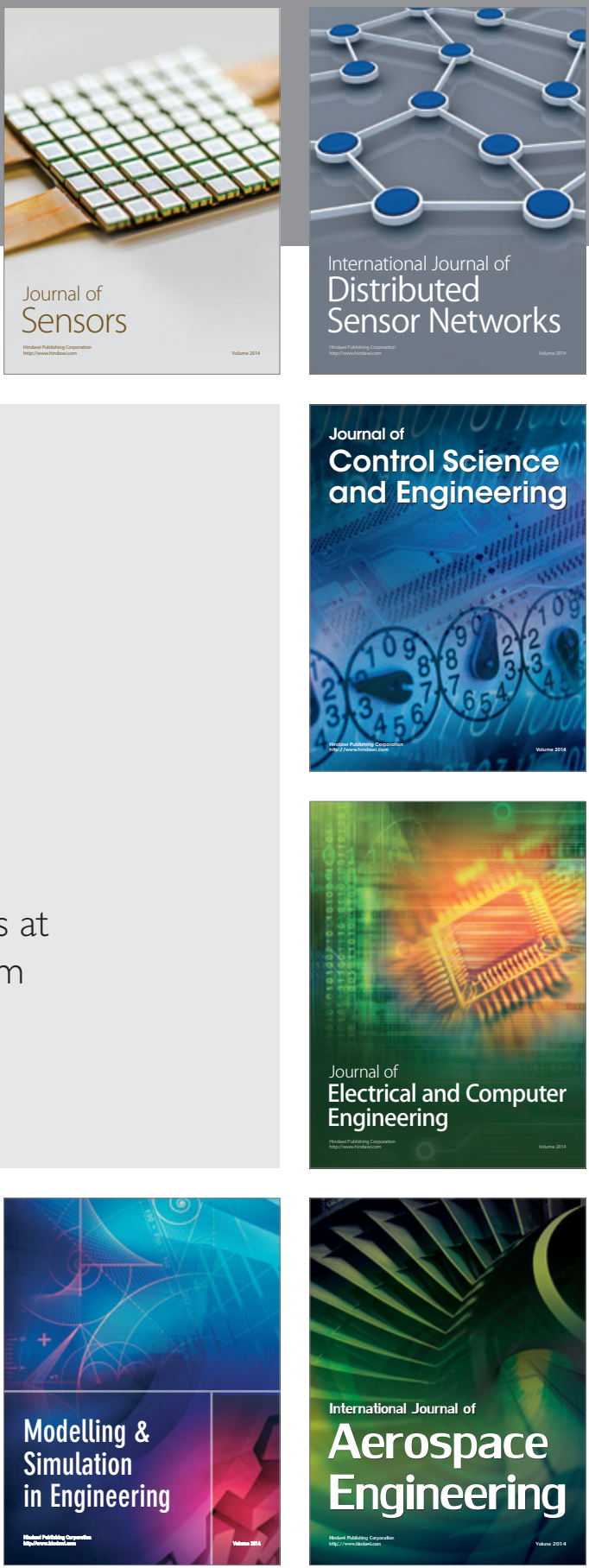

Journal of

Control Science

and Engineering
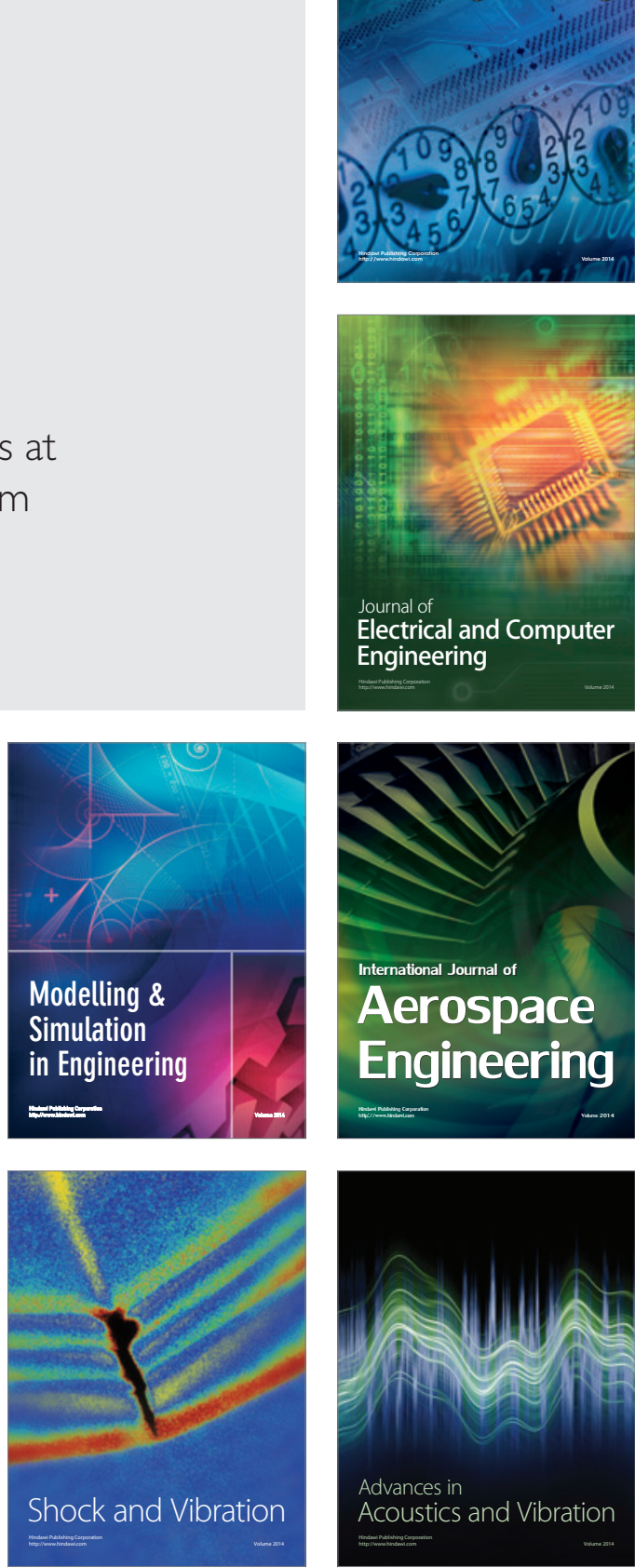